\title{
Aberration-corrected scanning transmission electron microscopy in single-atom catalysis: Probing the catalytically active centers
}

\author{
Jingyue (Jimmy) Liu a,b,*
}

\section{Introduction}

Human beings have been fascinated by the world of small. The use of magnifying glasses to "see" small creatures/objects that are beyond the resolution of our naked eyes led to the development of optical microscopes which revolutionized our understanding of the micro-world. The discovery of electrons, which possess the properties of both particles and waves, and the subsequent invention of the electron microscope (EM) by Ruska and Knoll in the early 1930s opened the door to the wonderful micro- and nano-world of matter. The ability to visualize and analyze individual single atoms, the fundamental building blocks of all matter, has been the ultimate goal for many generations of researchers. The resolving power of the early EMs, however, was orders of magnitude away from imaging individual single atoms. To achieve the goal of "seeing" single atoms required significant progress on improving electron sources and optics, reducing lens aberrations, improving microscope stability, and so on. The development of the cold-field emission electron gun by Crewe et al. in the early 1970s for constructing a sub-nanometer electron probe in a scanning transmission EM (STEM) made it possible to obtain the first electron microscopy images of single uranium atoms [1]. High-energy electrons scattered beyond the convergence angle of the incident electron beam were collected to form images of individual heavy metal atoms, either supported on ultrathin amorphous carbon films or decorated biological molecules. Such an imaging mode, denoted as annular dark-field (ADF) microscopy, was used to image even single silver atoms and atomic planes in small crystallites of uranium/thorium [2]. Electron-beam-induced dynamic changes of supported single atoms were investigated as well [3]. When such an ADF imaging method was applied to observing supported metal catalysts major issues were encountered: The contrast of ADF images depends on both the atomic number of the metal particles and the crystallographic orientation of the metal particles as well as the supporting crystallites. The presence of electron diffraction contrast in STEM-ADF images of supported metal catalysts makes it impossible to unambiguously and/or reliably distin- guish small metal particles/clusters from those of the support crystallites. Howie's group at Cambridge University proposed to increase the inner collection angle of the STEM-ADF detector to suppress the diffraction contrast of crystalline materials and thus recover the atomic number contrast of the supported noble metal particles/clusters [4,5]. The new imaging mode, denoted as high-angle ADF (HAADF), proved to be extremely valuable for characterizing supported metal catalysts [6-11].

Imaging of single metal atoms in supported metal catalysts had been a major challenge prior to the era of aberration-corrected STEMs. Rice et al. [7] reported in 1990 the direct imaging of single Pt atoms in zeolite frame works. Concurrently, Liu and Cowley [8] examined the presence of small Pt clusters/atoms in $\mathrm{Pt} /$ carbon, $\mathrm{Pt} / \gamma$-alumina and $\mathrm{Pt} / \mathrm{NaY}$ zeolite catalysts. By using a $300 \mathrm{kV}$ STEM instrument and with improved microscope stability, Nellist and Pennycook reported in 1996 the direct imaging of individual Pt atoms dispersed onto the insulating surfaces of a $\gamma-\mathrm{Al}_{2} \mathrm{O}_{3}$ support [12]. In the late $1990 \mathrm{~s}$ and early 2000s, the present author frequently observed the presence of single metal atoms in various types of industrial supported metal and alloy catalysts. All these observations, however, only demonstrated the capability of using the STEM-HAADF technique to visualize single metal atoms under specific or ideal imaging conditions. Routine examinations of single metal atoms in practical catalysts and the correlation of the observed single metal atoms to catalytic performances were not possible. Therefore, although single-atom imaging was demonstrated in the 1990s this imaging technique was not reliable enough for providing statistically meaningful information on correlating the presence of single metal atoms to the catalytic performance of supported metal catalysts $[9,10]$.

In this perspective, we will focus on discussing how to utilize aberration-corrected STEM-HAADF (ac-STEM-HAADF) for routinely examining, in supported metal catalysts, the presence of single metal atoms, determining their spatial distribution and dispersion, and evaluating their crystallographic relationship with respect to the surface structure of the support material. The ac-STEM-HAADF technique has proved to be indispensable for developing supported single-atom catalysts 
(SACs), especially for optimizing the catalyst synthesis protocols, and for understanding the fundamental mechanisms of single-atom catalysis [13-21]. The atomic scale data, acquired via ac-STEM imaging, on the coordination of single metal atoms on the surfaces of a support crystal can be directly used to establish models for DFT calculations of the catalytic processes of supported single metal atoms [13].

Although we focus, in this perspective, on the application of ac-STEM techniques to developing and understanding SACs one should keep in mind that the coupling of the ac-STEM with other characterization techniques such as extended X-ray absorption fine structure (EXAFS) spectroscopy, X-ray absorption near edge structure (XANES) spectroscopy, and Fourier transform infrared spectroscopy (FTIR) is extremely useful and powerful for characterizing the structure and understanding the catalytic properties of SACs [22,23].

\section{Imaging supported single metal atoms with aberration-corrected STEM}

The breakthroughs in correcting lens-aberrations in the late 1990s made it possible to significantly improve the spatial resolution of a conventional transmission electron microscope (CTEM) or STEM [24-27]. With the achievement of such aberration corrections, the sensitivity of single-atom imaging in the STEM is significantly improved: The chemical nature of all non-overlapping atoms in two-dimensional materials can be identified by quantitative image analysis [28] and heavy metal atoms in practical catalysts can be routinely examined with excellent image contrast $[11,13,21]$. Statistically meaningful and reliable data on the spatial distribution and dispersion of supported single metal atoms can be effectively utilized to understand the structure of supported metal catalysts and help optimizing the synthesis processes. Fig. 1, an ac-STEM-HAADF image of a $\mathrm{ZnO}$ supported Pt catalyst which was prepared by a wet chemistry method, clearly demonstrates the power of the modern ac-STEM technique in characterizing supported metal catalysts, especially supported noble metal catalysts. The spatial distribution, shape configuration, the crystallographic structure of Pt nanoparticles, clusters, and single atoms, and the crystal structure of the polycrystalline $\mathrm{ZnO}$ support are all clearly revealed and can be meaningfully analyzed. Correct interpretation of such images may provide useful information on correlating the catalyst structure to its performance (activity, selectivity and stability). For example, detailed analyses of Fig. 1 suggest that most of the isolated Pt atoms are located at the cation positions on the various surfaces of the $\mathrm{ZnO}$ support, a consequence of strong metal-support interactions. Some of the Pt clusters are highly disordered while others form well-defined facets with an epitaxial relationship with the corresponding ZnO nanocrystallites. The Pt clusters seem to preferentially grow at the grain boundaries or triple junctions of the polycrystalline ZnO support and some Pt clusters are crystallographically distorted to accommodate the strain field imposed by the lattice mismatch of the various crystal planes. The ac-STEM-HAADF technique provides unparalleled information on the atomic structure and configuration of supported metal

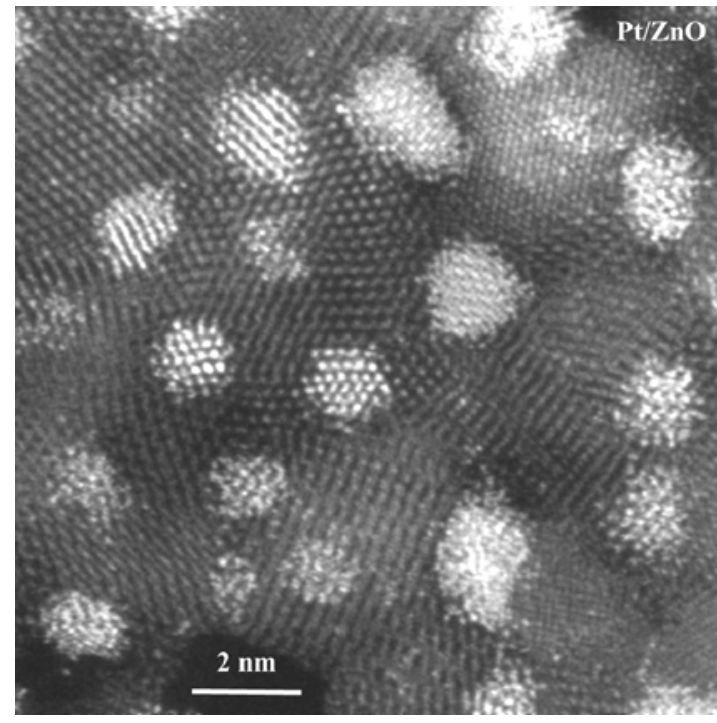

Fig. 1. Aberration-corrected STEM-HAADF image of a $\mathrm{ZnO}$ supported $\mathrm{Pt}$ catalyst clearly reveals the presence of Pt single atoms, clusters, and faceted nanoparticles. Some of the Pt clusters seem to grow epitaxially onto the support surface while others seem to be highly disordered. Most of the isolated $\mathrm{Pt}$ atoms are located at the cation positions of the various surfaces of the $\mathrm{ZnO}$ support.

nanoparticles, clusters, and even single atoms. Such atomic resolution images of supported metal catalysts convincingly demonstrate the structural complexity of supported metal catalysts: It is extremely difficult, if not impossible, to quantify the configurations of the huge numbers of metal clusters/particles in a supported metal catalyst. Such complexity in defining and evaluating statistically meaningful structural data poses a grand challenge to understanding the synthesis-structure-performance relationships of supported metal catalysts. For SACs, since we deal with only isolated single metal atoms such structural complexity is significantly reduced, especially if the support surface is structurally well-defined.

We started specifically synthesizing and characterizing supported single metal atoms in the mid to late 2000 s and reported the results at catalysis and microscopy conferences in a series of proceedings publications [29]. For example, we reported, in 2007, the results on strong anchoring of $\mathrm{Au}$ monomers and clusters onto $\mathrm{TiO}_{2}$ support surfaces (Fig. 2(a)) and Pt single atoms attached to carbon supports, and discussed the use of atomic resolution ac-STEM-HAADF technique to characterize supported metal catalysts [29a]. We discussed the anchoring mechanisms and emphasized processes to stabilize metal clusters and monomers. In 2008, we reported the synthesis and characterization of Pd monomers strongly anchored onto $\mathrm{ZnO}$ nanobelts and nanowires (Fig. 2(b)) and conducted detailed structural analyses (Fig. 2(c) and (d)) to reveal that the $\mathrm{Pd}$ monomers were located on the exact $\mathrm{Zn}$ cation positions of the $\mathrm{ZnO}$ nanobelts and nanowires [29b]. We further demonstrated that $\mathrm{Pd}$ single atoms grew along the rows of $\mathrm{Zn}$ cations to form short segments of adsorbed Pd rows or monolayer patches on the $\mathrm{ZnO}\{10-10\}$ nanoscale facets [29c]. High number-density Pd single atoms supported on $\mathrm{ZnO}$ nanowires were synthesized and characterized in 2009 and were tested for 

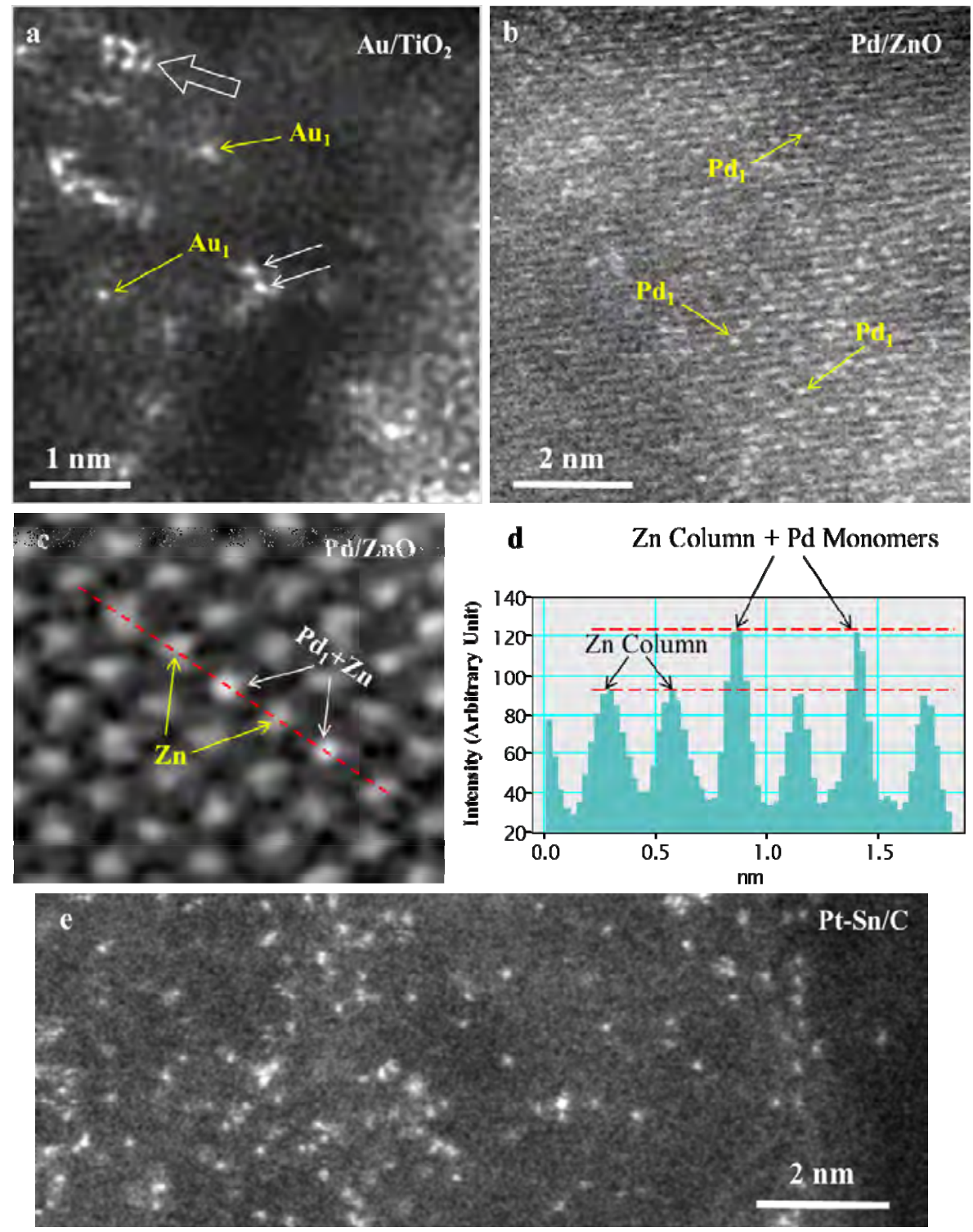

Fig. 2. STEM-HAADF images of $\mathrm{Au} / \mathrm{TiO}_{2}(\mathrm{a})$ and $\mathrm{Pd}_{1} / \mathrm{ZnO}$ nanowire (b) catalysts clearly reveal the single metal atoms on the surfaces of the supports. Magnified image (c) and intensity line scan (d) across a row of Zn atoms unambiguously show that the $\mathrm{Pd}_{1}$ atoms occupy exactly the $\mathrm{Zn}$ positions of the ZnO nanowire support. STEM-HAADF image of a Pt-Sn/C catalyst (e) shows the dispersion and spatial distribution of the metal atoms (The brighter dots represent Pt atoms and the grey dots represent Sn atoms). Adapted from Ref. [29].

steam reforming of methanol [29d]. We further reported in both 2008 and 2009 [29e] the synthesis and characterization of atomically dispersed Pt-Sn complexes, investigated the anchoring of single Pt atoms onto the defect sites of the XC-72 graphitic carbon powders (Fig. 2(e)), and studied how the isolated metal monomers and multimers grew, under in situ thermal treatment, into clusters and faceted nanoparticles. In 2010 and 2011, we investigated the challenges in ac-STEM-HAADF imaging of single metal atoms which are adsorbed on or embedded into the surfaces of catalyst supports. In all these studies, the ac-STEM-HAADF images unambiguously demonstrated the anchoring of isolated single metal atoms onto the surface defect sites of the support materials. Some of the work on ac-STEM-HAADF imaging of supported single metal atoms were summarized in a review paper on metal-support interactions in supported metal catalysts [11].
Allard et al. [30] reported in 2009 the ac-STEM study of a series of catalysts derived by $\mathrm{NaCN}$ solution leaching a parent 2 at. $\% \mathrm{Au} / \mathrm{Fe}_{2} \mathrm{O}_{3}$ catalyst (World Gold Council reference number 60C) prepared by the co-precipitation method and treated by calcination at $400{ }^{\circ} \mathrm{C}$. An extensive in situ heating study was conducted and they found a remarkable stability of $\mathrm{Au}$ atoms with redox treatments up to $400{ }^{\circ} \mathrm{C}$ and that $\mathrm{Au}$ atoms decorated step surfaces of the $\mathrm{Fe}_{2} \mathrm{O}_{3}$ support. Gold species internal to the $\mathrm{Fe}_{2} \mathrm{O}_{3}$ support material, however, was also observed to diffuse to the support surface and coalesced into discrete $\mathrm{Au}$ nanocrystals. Catalytic tests demonstrated that these cationic $\mathrm{Au}$ atoms supported on metal oxides yielded excellent activity for water-gas-shift and reforming reactions [31].

\section{Evaluating the dispersion of supported single metal atoms and improving synthesis protocols}


By late 2000s, it was undoubtedly proved that ac-STEM-HAADF technique could be routinely utilized to examine single metal atoms in practical supported noble metal catalysts with atomic resolution and excellent image contrast [11]. With such characterization tools readily available it became possible to unambiguously determine if one could directly synthesize supported metal catalysts that contain only isolated metal atoms dispersed on high-surface-area supports. Working together with Professor Tao Zhang's group at the Dalian Institute of Chemical Physics of the Chinese Academy of Sciences, we initiated, in 2009, a project of directly synthesizing supported single metal atoms by using a facile and scalable co-impregnation method but keeping the Pt loading levels $<0.2$ wt.\%, significantly lower than the normal metal loading levels of supported metal catalysts, to prepare $\mathrm{Pt} / \mathrm{FeO}_{x}$ catalysts. By late 2009, ac-STEM-HAADF images of the as-synthesized $\mathrm{Pt} / \mathrm{FeO}_{x}$ catalyst unambiguously confirmed that these new catalysts consisted of only isolated individual Pt atoms and no Pt clusters or particles were detected. The project team further optimized the synthesis protocols and was able to repeatedly and reliably synthesize $\mathrm{Pt}_{1} / \mathrm{FeO}_{x} \mathrm{SACs}$. Data from other charac- terization techniques (e.g., EXAFS, IR, XRD, and so on.) corroborated the conclusion reached by the ac-STEM-HAADF imaging technique that these newly synthesized low Pt loading catalysts consisted of only single Pt atoms. Catalytic evaluations of the synthesized $\mathrm{Pt}_{1} / \mathrm{FeO}_{x}$ SACs yielded excellent performance for CO oxidation and preferential oxidation (PROX) of CO in hydrogen-rich stream [13]. This systematic investigation established, for the first time, that SACs can be directly synthesized by facile and scalable wet chemistry methods and that supported single metal atoms mediate catalytic reactions. Subsequent density functional theory (DFT) calculations, with coordinates of the Pt single atoms derived from ac-STEM-HAADF images, provided the fundamental understanding of the catalytic cycles of the $\mathrm{Pt}_{1} / \mathrm{FeO}_{x}$ SACs [13].

In practice, one needs to use an integrated electron microscopy approach to fully characterize the spatial distribution and dispersion of a supported metal catalyst [11,21]. When the metal loading levels become very low the presence of any large metal particles can significantly deviate the correct correlation of the observed catalyst structure to the catalytic performance. Fig. 3(a)-(d) show a set of electron microscopy images of a
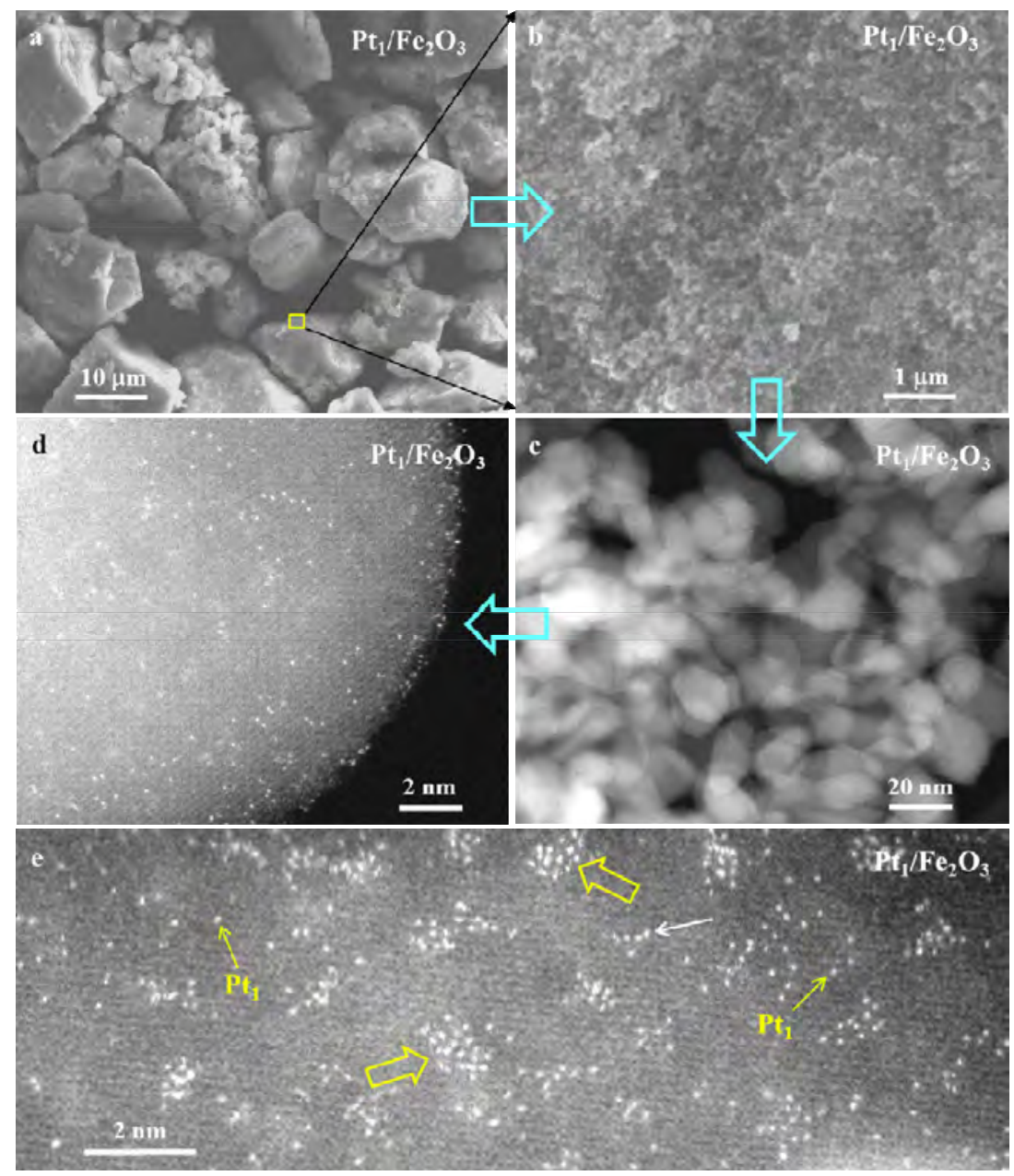

Fig. 3. SEM images (a,b) and STEM-HAADF images (c-e) of a highly loaded $\mathrm{Pt}_{1} / \mathrm{Fe}_{2} \mathrm{O}_{3}$ SAC prepared by adsorption of Pt salt precursor species onto pre-formed $\mathrm{Fe}_{2} \mathrm{O}_{3}$ crystallites. This set of images confirms that the as-synthesized $\mathrm{Pt}_{1} / \mathrm{Fe}_{2} \mathrm{O}_{3} \mathrm{SAC}$ does not contain any Pt particles or clusters and contains only isolated $\mathrm{Pt}_{1}$ atoms anchored onto the surfaces of the $\mathrm{Fe}_{2} \mathrm{O}_{3}$ crystallites. The block arrows in Fig. 3(e) indicate the appearance of loosely connected Pt "clusters". It is not fully understood why such "clusters" were formed during the synthesis processes. 
$\mathrm{Pt}_{1} / \mathrm{Fe}_{2} \mathrm{O}_{3}$ SAC with different magnifications to confirm the absence of any sizes of Pt particles or clusters and the presence of only isolated single Pt atoms. The examination of supported metal catalysts by secondary/backscattered electron imaging in a modern field-emission gun SEM and low magnification STEM-HAADF imaging is critical to optimizing the synthesis protocols of supported metal catalysts, especially SACs. High magnification, atomic resolution ac-STEM-HAADF images (e.g., Fig. 3(d)) can provide direct evidence of the dispersion and spatial distribution of metal atoms in the synthesized SACs. By examining such set of images of the various regions of the catalysts one should be able to quickly diagnose if the as-prepared catalysts are SACs.

Compared to the broad beam characterization techniques such as EXAFS or IR the power of electron microscopy is to provide spatially resolved local information. Such imaging technique is especially valuable for characterizing heterogeneous samples which may possess various levels of heterogeneity from micrometer scale down to nanometer or atomic scale. Fig. 3(e), an ac-STEM-HAADF image of a highly loaded $\mathrm{Pt}_{1} / \mathrm{Fe}_{2} \mathrm{O}_{3}$ SAC which was prepared by a strong adsorption method and was calcined in air, clearly demonstrates the power of direct atomic resolution imaging of SACs. Although all the Pt atoms are atomically dispersed the image clearly reveals a variety of configurational heterogeneity of the Pt single atoms: Isolated $\mathrm{Pt}_{1}$ (e.g., those indicated by the yellow arrows), various types of loosely connected and atomically dispersed Pt "clusters" (e.g., those indicated by the block yellow arrows), and other types of Pt configurations (e.g., indicated by the white arrow) were all present in the as-synthesized $\mathrm{Pt}_{1} / \mathrm{Fe}_{2} \mathrm{O}_{3} \mathrm{SAC}$.

The co-existence of many configurations of loosely connected "clusters" of Pt atoms on the $\mathrm{Fe}_{2} \mathrm{O}_{3}$ support surfaces is difficult to understand since the distances among the individual Pt atoms in these "clusters" are much larger than those of pure Pt metal clusters. Since the $\mathrm{Pt}_{1} / \mathrm{Fe}_{2} \mathrm{O}_{3} \mathrm{SAC}$ was prepared by a strong solution adsorption method and was calcined at a relatively high temperature the Pt atoms can be viewed as contrast enhancing decorations of surface defects or functional groups. The various "patterns" of the Pt atoms shown in Fig. 3(e) may suggest the presence of various types of anchoring sites-surface defects or functional groups. The heterogeneous nature of the support surfaces and functional groups defines the various configurations or "patterns" of the individual $\mathrm{Pt}$ atoms observed in ac-STEM-HAADF images. It is not clear at all how these various configurations of individual Pt atoms were formed or how they may affect the catalytic properties of SACs. When the metal loading level is low such configurations or patters were rarely observed. From this perspective, high metal loading SACs may possess various types of catalytically active sites since the single metal atoms may probably be stabilized by different types of anchoring sites which are present on the support surfaces.

4. Determining the coordinates of supported single metal atoms and the catalytically active centers of single-atom catalysts

\subsection{Crystalline supports}

The ac-STEM-HAADF images not only provide spatial distribution and dispersion of supported metal atoms but also can provide the spatial coordination of each individual $\mathrm{Pt}$ atoms with respect to the surface atomic structure of the support material provided that the support is crystalline in nature. By simultaneously imaging the atomic structure of the support, which should be oriented close to a crystallographic zone axis, and the supported single metal atoms one can unambiguously identify the exact lateral coordinates of the supported single metal atoms with respect to the surface crystal structure of the support. Fig. 4(a) demonstrates an example of such characterization of a $\mathrm{Pt}_{1} / \mathrm{Fe}_{2} \mathrm{O}_{3} \mathrm{SAC}$ which was prepared by a strong solution adsorption method. Fig. 4(b) shows the surface plot profile of Fig. 4(a), clearly revealing the differences in image intensity between the $\left(\mathrm{Pt}_{1}+\mathrm{Fe}\right.$ column) and its neighboring Fe columns. The oxygen atoms could not be detected in the STEM-HAADF imaging mode unless the sample is extremely thin. Both Fig. 4(a) and (b) directly and undoubtedly confirm that the $\mathrm{Pt}_{1}$ atoms occupy exactly the Fe cation positions. Therefore, the lateral coordinates of the $\mathrm{Pt}_{1}$ atoms can be determined. The information on the coordinate of the $\mathrm{Pt}_{1}$ atoms along the incident electron beam direction (the $z$-axis), however, cannot be precisely determined since the images are two-dimensional projections of the sample along the incident electron beam direction. By changing the lens focus value one can conduct an "optical sectioning" of the sample to estimate the location of the $\mathrm{Pt}_{1}$ atoms. With detailed image simulations and quantitative image intensity analysis one can, in theory, determine the depth location of the $\mathrm{Pt}_{1}$ atoms. In practice, however, one can only estimate the $z$-coordinate of the $\mathrm{Pt}_{1}$ atoms with an accuracy of about 1-2 nm. Unless in special cases (c.f. Fig. 8) we need other supplementary information to determine the exact $z$-coordinate of the metal single atoms. By examining the samples in cross-sectional configuration (e.g., ultramicrotoming the embedded catalyst powders into ultrathin slices) one can also extract useful information on the location of the adsorbed metal atoms. Such an approach works well for heavy-element metal atoms supported on light-element nanostructures.

Since the $\mathrm{Pt}_{1} / \mathrm{Fe}_{2} \mathrm{O}_{3} \mathrm{SAC}$ was prepared by a strong solution adsorption method the $\mathrm{Pt}_{1}$ atoms should be most probably located on the surfaces of the $\mathrm{Fe}_{2} \mathrm{O}_{3}$ crystallites, not within the support. The STEM-HAADF image of Fig. 4(a) then suggests two possibilities: (1) the $\mathrm{Pt}_{1}$ atoms are anchored to the surface $\mathrm{Fe}$ cation vacancy sites-surface doping and (2) the $\mathrm{Pt}_{1}$ atoms are located at the $\mathrm{Fe}$ cation positions above the $\mathrm{Fe}_{2} \mathrm{O}_{3}$ surface and are coordinated with the oxygen atoms of the $\mathrm{Fe}_{2} \mathrm{O}_{3}$ surface-epitaxial adsorption.

Since both the chemical nature and the size of the Pt atoms are different from those of the Fe atoms geometric distortions in both the surface doping or adsorption configurations may occur. In either case, strong metal-support interactions can induce charge transfer and the anchoring of the $\mathrm{Pt}_{1}$ atoms, to the first order approximation, is due to bonding between the $\mathrm{Pt}_{1}$ atoms and the oxygen anions of the $\mathrm{Fe}_{2} \mathrm{O}_{3}$ surfaces. The surface doped $\mathrm{Pt}_{1}$ atoms should be anchored stronger than the 

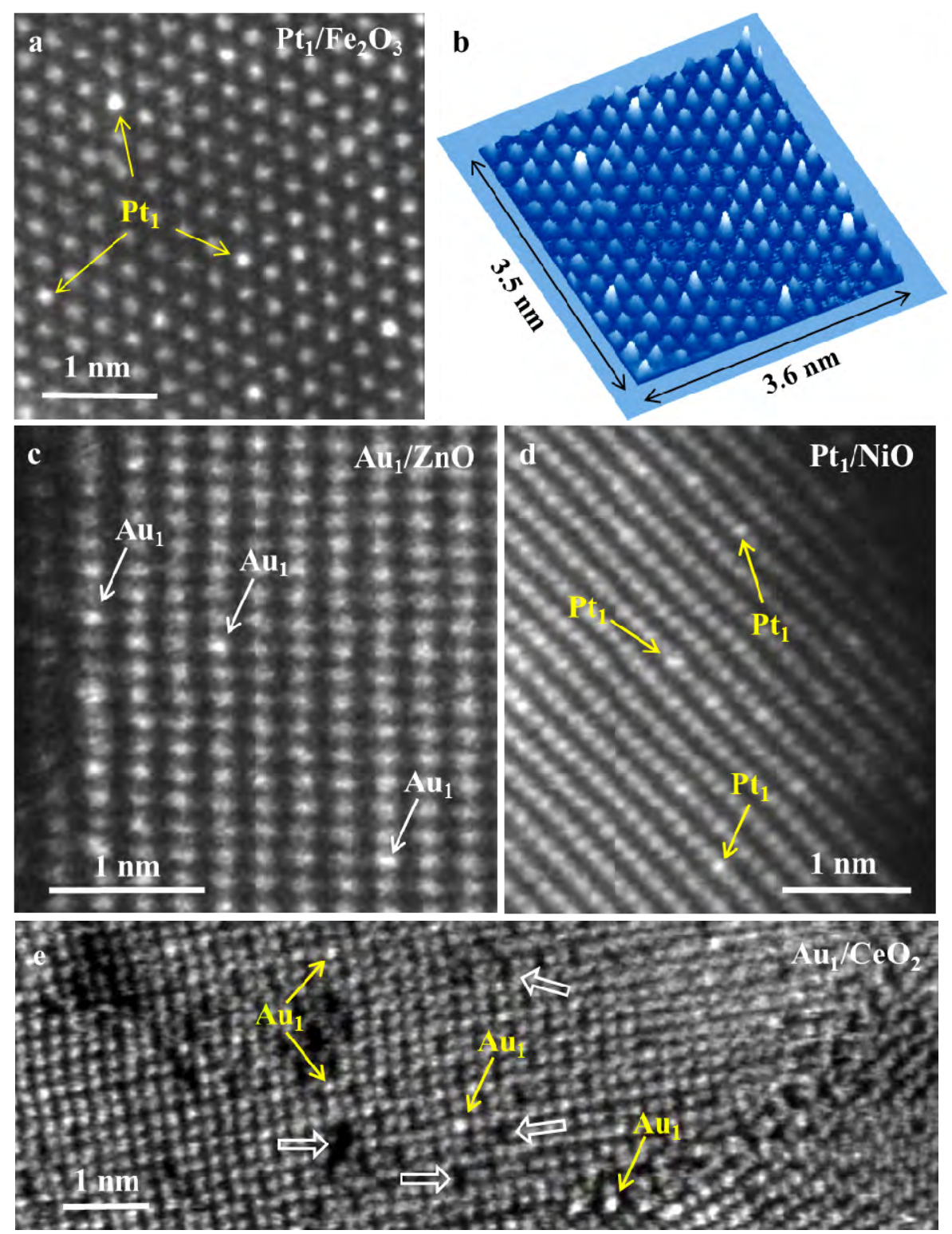

Fig. 4. Aberration-corrected STEM-HAADF images (a,c,d,e) of Pt and Au single atoms dispersed onto $\mathrm{Fe}_{2} \mathrm{O}_{3}(\mathrm{a}), \mathrm{ZnO}$ nanowire (c), $\mathrm{NiO}(\mathrm{d})$ and $\mathrm{CeO} \mathrm{O}_{2}(\mathrm{e})$ support clearly show that the isolated $\mathrm{Pt}_{1}$ and $\mathrm{Au}_{1}$ atoms occupy exactly the cation positions of the metal oxide support. Figure $4 \mathrm{~b}$ displays the intensity surface plot of Fig. 4(a) to demonstrate the image intensity of the $\mathrm{Pt}_{1}$ atoms and their positional relationship with respect to the Fe cation positions. The white block arrows in Fig. 4(e) indicate Ce vacancy sites or clusters of Ce vacancies. Adapted from Ref. [21].

surface adsorbed $\mathrm{Pt}_{1}$ atoms. With extremely low levels of metal loading and with appropriate calcination treatments the single metal atoms generally occupy the surface cation vacancy sites of a reducible metal oxide support. Those metal oxide supports which possess a large number of cation vacancies (e.g., ceria) may be able to accommodate high levels of metal loading and maintain the characteristics of stable SACs $[13,15,19]$.

Such a cation vacancy anchoring mechanism seems to be general for metal oxide supported single metal atoms, especially single noble metal atoms on reducible metal oxides. Fig. 4(c) and (d) shows, respectively, ac-STEM-HAADF images of a $\mathrm{Au}_{1} / \mathrm{ZnO}$ and $\mathrm{a} \mathrm{Pt}_{1} / \mathrm{NiO} \mathrm{SAC}$, prepared by strong adsorption method, clearly revealing the lateral coordinates of the supported $\mathrm{Au}_{1}$ and $\mathrm{Pt}_{1}$ single atoms. We still do not know, during the wet chemistry preparation processes and the subsequent calcination process, how these single metal atoms incorporate themselves into the surface cation vacancy positions of the metal oxides. Our experiences show that some metal oxides such as $\mathrm{NiO}$ (Fig. 4(d)) and $\mathrm{CeO}_{2}$ (Fig. 4(e)) can accommodate high levels of single metal atoms. When $\mathrm{CeO}_{2}$ was prepared via a wet chemistry method, Ce vacancy sites or even clusters of Ce vacancies can be directly visualized in ac-STEM-HAADF images (e.g., Fig. 4(e)) [32]. The stability of the supported metal SACs depends on the nature of the single metal atoms, the surface structure and chemistry of the support material, and the degree of metal-support interactions. The presence of surface functional groups such as hydroxyl groups can significantly modify the anchoring of the supported single metal atoms. Sub-surface 
defects and other types of bulk defects may also influence the stability of the adsorbed single metal atoms $[33,34]$.

With the position of the supported $\mathrm{M}_{1}$ atoms defined we can discuss the potential active centers. The newly formed entity $\left(M_{1}-O_{x^{-}}\right)$, where $M_{1}$ represent the single metal atom and $x$ represents the number of the oxygen anions of the support which are bonded to the single metal atom, plays a major role in determining the catalytic properties of the supported metal SACs [13]. Depending on the specific location (with respect to the crystal surface structure of the support) of the $\mathrm{M}_{1}$ atom the adsorption property of the newly formed $\left(M_{1}-O_{x^{-}}\right)$species can be different. Therefore, supported metal SACs can be considered to be structure sensitive. More experimental results are needed to confirm the structure sensitivity of SACs for various catalytic reactions.

When the charge transfer between the supported single metal atom and the support is nonlocal then the entity $\left(M_{1}-O_{x}-\mathrm{M}_{y} S_{-}\right)$, where $y$ represents the number of the nearest cations that are bonded to the $x$ oxygen anions of the support and $s$ represents the support, may need to be considered. In this case, the redox property of the support surface may exert significant effects on the catalytic property of the supported metal SAC. When the single metal atom is supported on the surface of another metal the charge transfer can be completely delocalized and the electronic band structure of the support surface may be modified. The major electronic modification in this case is primarily that of the supported single metal atom and the consequence of the metal-support interaction is to modify the adsorption properties of the single metal atom [35].

The above proposed model of the SACs may not work if the metal loading levels are higher than what the support cation vacancies can accommodate. The next potential anchoring sites could probably be at the positions where the next layer of the support cations could take. Other types of defects on the support surfaces such as steps, kinks, corners, dislocations, etc. could become the anchoring sites as well. We do not, however, have feasible techniques to directly imaging these defects in practical supported metal catalysts. More research is needed to understand how the various surface defects can anchor single metal atoms. With increasing levels of metal loading, the distribution and dispersion of the single metal atoms become more complicated (cf. Fig. 3(e)). Our recent experimental results demonstrate that surface steps on 2D metal oxides supports can strongly anchor noble metal atoms.

When surface functional groups are present on a support surface then the interactions between the supported single metal atoms with the surface functional groups may become dominant and thus weaken the metal-support interaction. The newly formed complexes $\left(\mathrm{M}_{1}\right.$ atom plus the surface functional group) can function as catalytically active centers. For example, Flytzani-Stephanopoulos' group has published reports on developing supported $\mathrm{Pt}(\mathrm{II})-\mathrm{O}_{x}(\mathrm{OH})_{y^{-}}$and $\mathrm{Au}(\mathrm{I})-\mathrm{O}_{x}(\mathrm{OH})_{y^{-}}$complexes for the water-gas-shift reaction [16,20,31]. They also utilized alkali ions to stabilize supported single metal species to form $\mathrm{M}_{1}-\mathrm{O}_{x}-(\mathrm{OH})_{y}-\mathrm{Na}_{x}$ clusters which become the catalytically active centers [31,36]. Direct visualization of these cluster complexes have not yet been accomplished by electron mi- croscopy techniques primarily due to the electron beam induced effects and the weak scattering of electrons by light elements. Surface functional groups on supports can suppress the effects of the support on the catalytic performances of the supported SACs. When the supported single metal atoms interact strongly with the surface functional groups the structure and the chemistry of the support surfaces may become less relevant [37]. Under these conditions and for specific catalytic reactions the role of the support becomes a pure physical carrier of the catalytically active complexes [31] and the supported SAC may behave as a single site heterogeneous catalyst (SSHC) [38].

The atomic scale information on the coordinates of the supported single metal atoms with respect to the structure of the support surface is extremely useful for establishing models for DFT calculations to assess the stability of the $\mathrm{Pt}_{1}$ atoms, their electronic interactions with the support surfaces, and ultimately their catalytic processes [13]. Unlike for supported metal cluster or nanoparticle catalysts the definitive structural relationship of the $\mathrm{M}_{1}$ atom with the support surface structure of a SAC makes it possible to directly use the output of the ac-STEM-HAADF imaging for DFT calculations of the fundamental catalytic processes of SACs.

Although we used metal oxide supports as examples to illustrate the power of ac-STEM-HAADF technique in determining the catalytically active centers of the metal oxides supported SACs the discussions given above should apply to other types of crystalline supports as well. In particular, ac-STEM-HAADF, in combination with EXAFS technique, is extremely powerful and useful for characterizing the atomic structure of single metal atoms dispersed into zeolites or metal organic frameworks $[14,39,40]$. The ac-STEM-HAADF technique is ideal for characterizing single metal atoms or small clusters dispersed onto 2D materials such as graphene, transition-metal dichalcogenides, metal oxides, and so on. With 2D materials as supports even single atoms of light elements can be detected and in some cases chemically analyzed, and complete structural information of the support material can be determined [28,41-44].

\subsection{Non-crystalline supports}

When the support material is not crystalline then all the structural information of the support surface is lost and electron microscopy can only provide the spatial distribution and dispersion of the supported single metal atoms. Such supports include activated carbons, amorphous silica, and some highly disordered alumina. For example, Fig. 5(a) shows a ac-STEM-HAADF image of a $\mathrm{Pd}_{1} / \mathrm{C}$ SAC prepared by strong adsorption of metal precursor species onto highly activated mesoporous carbon supports and Fig. 5(b) shows a similar image of high number-density $\mathrm{Si}$ atoms deposited onto the same type of activated mesoporous carbon via a high temperature chemical vapor deposition process. Although the mesoporous carbon support is highly disordered and almost amorphous-like the ac-STEM-HAADF images clearly show the isolated individual $\mathrm{Pd}_{1}$ and $\mathrm{Si}_{1}$ single atoms. Furthermore, by examining the images carefully one can induce that both the $\mathrm{Pd}_{1}$ 

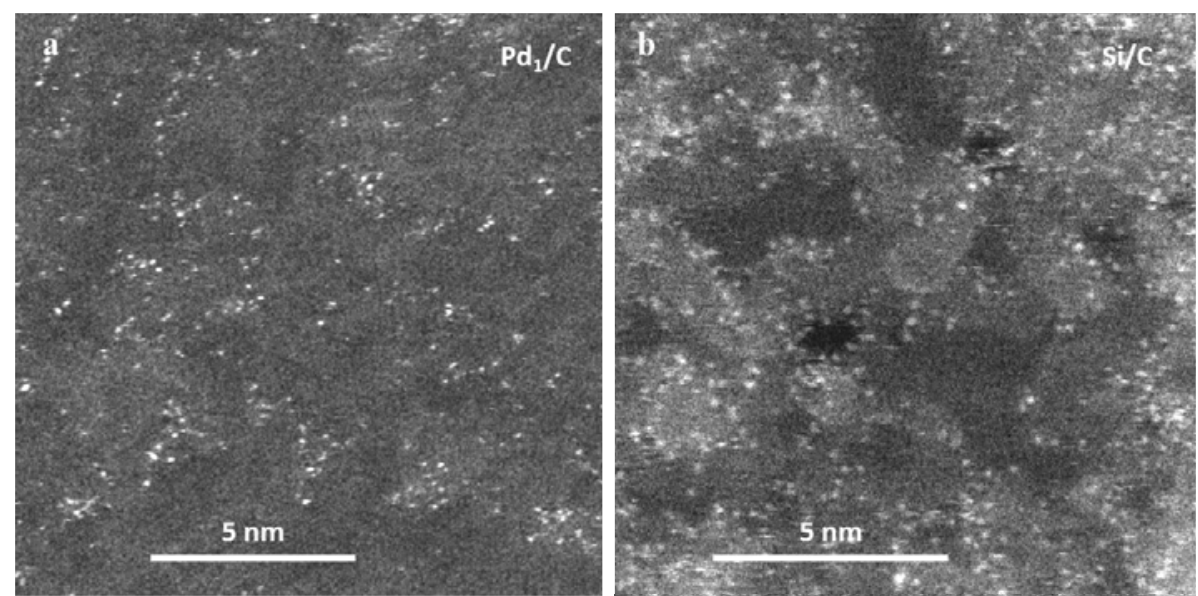

Fig. 5. Aberration-corrected STEM-HAADF images of single $\mathrm{Pd}_{1}(\mathrm{a})$ and $\mathrm{Si}_{1}$ (b) atoms dispersed onto activated mesoporous carbon supports. The Pd and $\mathrm{Si}$ atoms seem to decorate the edges of nanoscale graphene patches.

and $\mathrm{Si}_{1}$ atoms preferentially decorated the edges of small carbon patches (probably nanoscale graphene sheets). It is highly plausible that defects on, or edges of, graphene sheets acted as anchoring sites for the single atoms. The $\mathrm{Pd}_{1}$ atoms seem to have a tendency to be loosely clustered together while the (high-number density) Si atoms uniformly decorated the edges of the nanoscale carbon patches, suggesting stronger anchoring of the Si atoms to the carbon support. In fact, these Si atoms did not agglomerate at all even after prolonged annealing under $\mathrm{N}_{2}$ gas environment at temperatures above $1200^{\circ} \mathrm{C}$.

Charge transfer between the anchored single atoms and the carbon support can occur and strong interactions can result in chemical bonding. Such interactions will obviously modify the chemical and catalytic properties of the adsorbed single metal atoms. Compared to metal oxide supported SACs, however, the lack of well-defined structures of activated carbons or other types of amorphous support materials makes it difficult to provide the coordinates of the single metal atoms. Our extensive imaging experiences suggest that under electron beam irradiation the stability of these single metal atoms was different: some atoms moved constantly while others did not move at all, reflecting the various degree of anchoring, which in turn affects the catalytic properties of these single atoms.

For N-doped carbon materials, single metal atoms, for example, $\mathrm{Fe}$ or $\mathrm{Co}$, can strongly interact with the $\mathrm{N}$ dopants to form $\mathrm{M}_{1}-\mathrm{N}_{x}$ species that provide unique catalytic properties $[41,43,45,46]$. Such SACs have been synthesized for liquid-, electro-, and photo-catalytic reactions. Similarly, other types of dopants such as $\mathrm{O}, \mathrm{S}, \mathrm{P}$ or $\mathrm{Cl}$ can interact with $\mathrm{M}_{1}$ atoms to form anchored complexes which may possess unique adsorption or catalytic properties. Aberration-corrected EM may be able to provide valuable information on the catalytic processes of such catalytic systems provided that the electron beam induced effects can be significantly suppressed (see next section for discussions).

\section{Challenges and opportunities}

\subsection{Electron-beam induced effects}

When high-energy electrons interact with a sample both elastic and inelastic scattering processes occur. These energy and/or momentum transfer processes can significantly affect, depending on the nature of the sample, the structural and compositional integrity of the region of interest. Such electron-beam induced effects include knock-on damage which displaces an atom within a crystal or at its surface. The electron-beam induced motion of atoms are especially severe for adatoms on a support surface. Furthermore, under intense electron beam irradiation inside a high vacuum electron microscope surface functional groups can evaporate or be modified. The rate of the atom displacement depends on incident electron energy, the current density of the electron probe, the total accumulated dose, the atomic number of the adatoms, the binding strength between the adatoms and the support surface as well as the structure and the chemistry of the support material. Electron-beam induced damage of the catalyst support can become so severe that the support structure may collapse even before any meaningful structural information can be acquired. Such materials include zeolites and metal organic frameworks.

Fig. 6, a series of ac-STEM-HAADF images of a $\mathrm{Co}_{1} / \mathrm{N}$-doped carbon SAC, clearly demonstrates the severe effects of the 200 $\mathrm{keV}$ electron beam on the structural integrity of the loosely connected 12-atom Co cluster. Under electron beam irradiation, the configuration of the Co cluster changed continuously because of the severe damage of the $\mathrm{N}$-doped carbon support by the intense electron beam, resulting in the destabilization of the Co atoms. Furthermore, if the energy transfer from the electron beam to the Co atoms is higher than the binding energies of the Co atoms to the carbon support then the electron beam can destabilize the Co atoms or clusters. Metal oxide supports are generally susceptible to electron-beam induced effects such as electron-induced sputtering, ionization, and charging which can modify the integrity of the examined samples. Such electron-beam induced movement of adatoms poses a significant challenge for correctly identifying the true coordinates of the surface adsorbed metal atoms in SACs. Low dose methods and other types of practical imaging protocols should be followed in order to obtain the correct information on the 

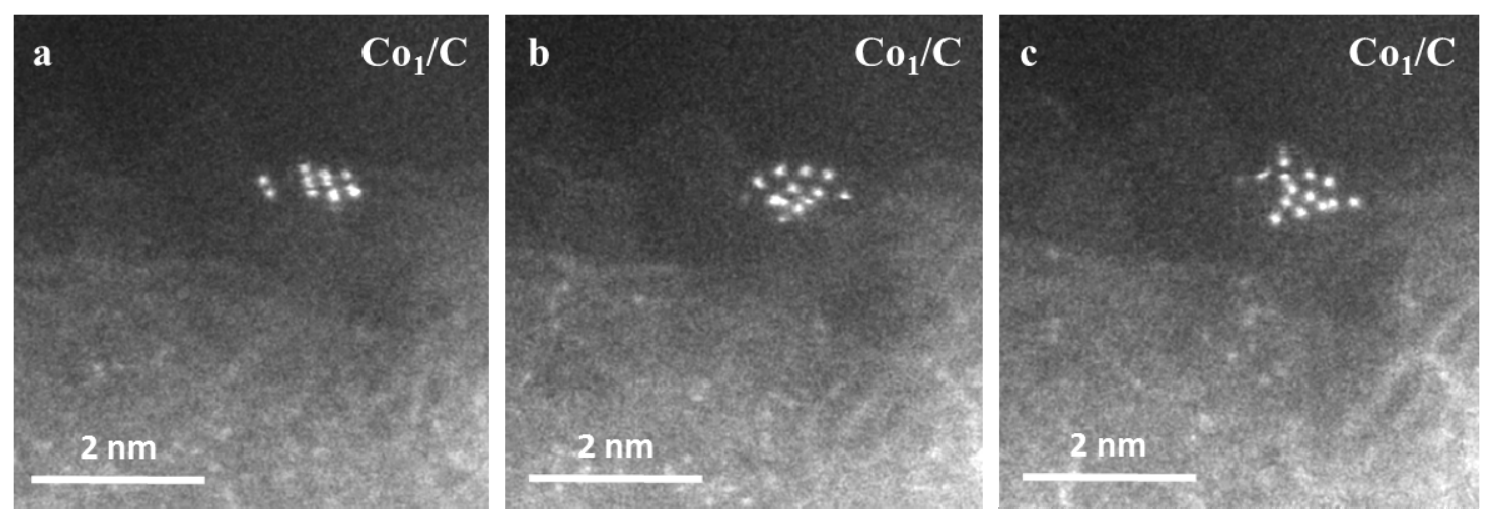

Fig. 6. Aberration-corrected STEM-HAADF images of a $\mathrm{Co}_{1} / \mathrm{C}$ SAC demonstrate the dynamic changes of the 12 -atom Co clusters under electron beam irradiation. Under the $200 \mathrm{keV}$ electron beam the Co cluster continuously changed its configuration probably due to the electron-beam induced damage of the carbon support material. Use of low energy electron beams may alleviate such electron-beam induced effects.

location of the adatoms on support surfaces.

In most cases, the electron-beam induced effects may increase with the sample temperature. Furthermore, high-energy electrons can ionize the gas molecules inside the sample chamber of an environmental EM or within the space of specialized sample holders that are filled with gases or liquids. High-energy electron induced effects pose the most critical barrier to the broad application of electron microscopy techniques to extracting structural information of a plethora of materials including catalytic materials. Unfortunately, we do not have a good understanding of the electron-beam induced effects and how to avoid these effects without compromising the capabilities of the modern electron microscopes. Significant progress in this field of research is urgently needed.

For most of the supported metal catalysts the use of lower electron beam energies may alleviate the electron-beam induced effects. For example, the use of incident electrons with energies $<60 \mathrm{keV}$ are preferred for characterizing carbon supported catalysts or for examining metal atoms supported on 2D materials. Investigations on the electron-beam induced motion of adatoms inside electron microscopes and the choice of selecting appropriate electron microscope operating voltages have been discussed $[47,48]$. The electron-beam induced effects should be investigated first before one can correctly interpret the images/spectra obtained in an environmental EM or by using gas or liquid sample holders.

\subsection{New developments}

A modern aberration-corrected electron microscope is generally equipped with detectors for collecting emitted characteristic X-rays and/or for analyzing inelastically scattered electrons to determine the composition or the electronic structure of the probed area of interest. The electron-beam induced effects, however, pose a serious obstacle to routinely identifying the nature of individual atoms dispersed on high-surface-area supports. When the single atoms are embedded into the surfaces of 2D supports or are located in the interior regions of a support both the energy dispersive X-ray spectroscopy (EDS) and electron energy loss spectroscopy (EELS) techniques can be used to identify the atomic species of the individual atoms and, in favorable cases, provide information on the oxidation states of the probed atoms [49-51]. The recent development of electron beam monochromators on ac-STEM instruments significantly improved the energy resolution achievable in STEM-EELS to $<10 \mathrm{meV}$ [52,53], comparable to the energy resolution of optical spectroscopy techniques. For an adsorbed metal atom on the surface of a support material the high intensity electron probe, which is required for generating statistically meaningful analytical signals, usually destabilizes the adsorbed metal atom and/or damages the support material. Therefore, routine analyses of supported single metal atoms by EDS and EELS techniques have not been realized yet. Highly efficient detectors are needed in order to accomplish the challenging goal of reliably extracting information on the electronic structure or oxidation state of a supported single metal atom.

The electron microscope usually provides a 2D projection image of a three-dimensional (3D) object. During such an imaging process, not only the 3D information of the sample is lost but also the interpretation of the projected image can become challenging. For mesoporous materials, this effect can pose a serious issue for determining the location of the metal atoms or clusters. For example, Fig. 7(a) and (b) show a pair of ac-STEM-HAADF images of the same area of a carbon supported Pt cluster catalyst but obtained with a different focus value of the probe-forming lens. The two images reveal Pt atoms and clusters located at the different depths within the activated carbon support. In this particular case, it is highly desirable to obtain 3D images of the sample so that the true spatial distribution of the Pt single atoms and clusters can be reliably analyzed. The recent advancement in high-resolution electron tomography makes it possible to obtain 3D information on particle size distributions in supported metal catalysts, the 3D shape of individual nanoparticles, the shape and connectivity of the support pore structures, and the 3D information on the particle-support interfaces [54-56]. Development of atomic resolution 3D imaging has been progressing rapidly but routine analyses by these techniques of practical supported metal catalysts have not been realized yet. Atomic resolution tomography will undoubtedly be critical to understanding the 3D distribution of 

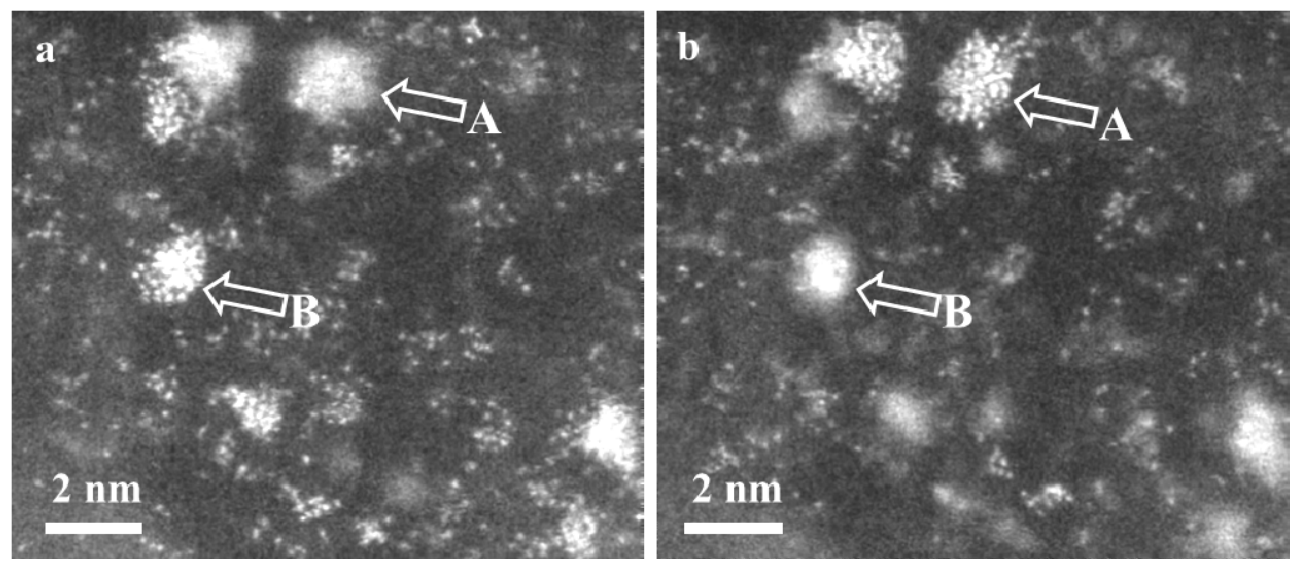

Fig. 7. Aberration-corrected STEM-HAADF images of the same area of a Pt/carbon catalyst demonstrate the need for 3D imaging of highly mesoporous support materials. By changing the lens focus, Pt single atoms and clusters located at different depths of the carbon support can be imaged. The same Pt clusters are indicated by A and B, respectively, in the corresponding images. The Pt atoms and small clusters uniformly distributed throughout the mesoporous carbon support.

the individual components of supported SACs and the structural/spatial relationships of the observed individual metal atoms with respect to the surface structure of the supports.

To develop robust SAC synthesis protocols that stabilize single metal atoms on appropriate supports requires a fundamental understanding of the dynamics of the supported single atoms under gas environments and at elevated temperatures. Recent technologies for in situ reaction studies utilizing a closed-cell MEMS-based reactor specimen holder in ac-STEM instrument have shown the capability of imaging atomic columns on metal particles at pressures up to an atmosphere and temperatures up to $1000{ }^{\circ} \mathrm{C}$ [57]. Direct tracking of the movement of single metal atoms during reaction experiments remains problematical, primarily due to the scattering phenomena relative to the elements of the gas cell geometry and electron-beam induced effects. Recent progress made it possible to observe single atom reactions on a $\mathrm{Pt}_{1} / \mathrm{NiO} \mathrm{SAC}$ [58]. Further development of this type of in situ or operando imaging techniques is expected to have a significant positive impact on the understanding of the stability of supported SACs and on developing robust practical SACs for desired catalytic reactions. Aberration-corrected environmental STEM has also been developed and investigations on the dynamic sintering behavior of supported single metal atoms and clusters under gas environment have been reported $[59,60]$.

Designing the support structure is as important as controlling the catalytically active components. For developing SACs, the nature of the support surfaces is the core of fabricating desired catalysts: Their structural and electronic properties control the anchoring, spatial distribution, and the charge transfer processes between the metal atoms and the support. With the rapid advancement in controlled synthesis of nanostructured metal oxides it is possible to directly determine not only the lateral coordinates of the supported metal atoms but also their structural relationship with the support surface in the third dimension. Fig. 8 illustrates such an example. The $\mathrm{Ir}_{1} / \mathrm{ZnO}$ nanowire catalyst was prepared by a strong solution adsorption method and was calcined at $500{ }^{\circ} \mathrm{C}$ for several

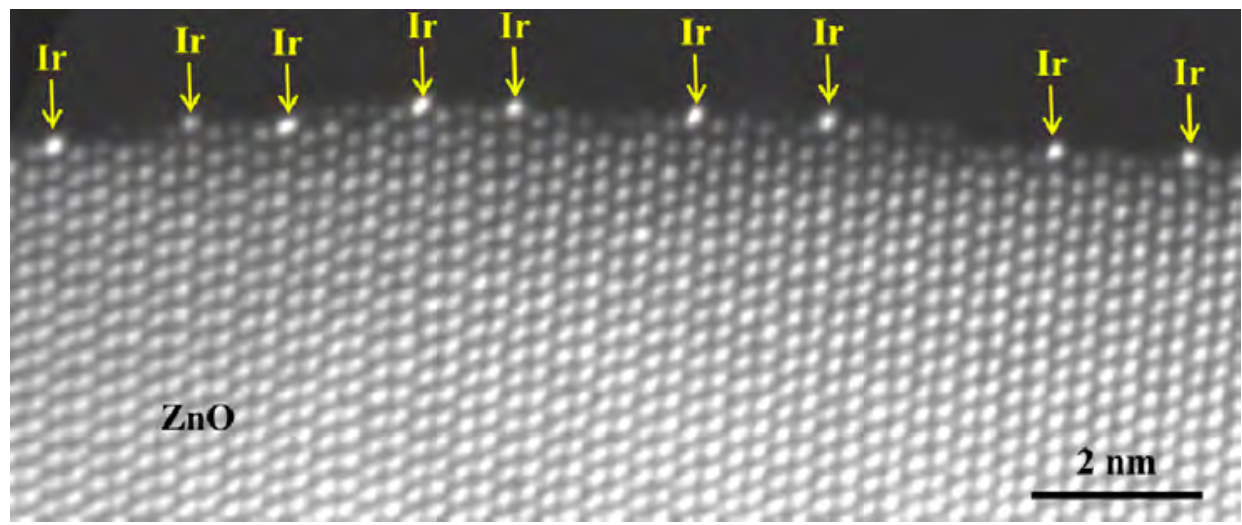

Fig. 8. Aberration-corrected STEM-HAADF profile image of an $\operatorname{Ir}_{1} / \mathrm{ZnO}$ nanowire catalyst clearly shows the location of the Ir atoms with respect to the $\mathrm{ZnO}\{10-10\}$ nanoscale facets: The Ir atoms were embedded into the $\mathrm{ZnO}\{10-10\}$ surface as if the Ir atoms replaced the $\mathrm{Zn}$ atoms. In such profile images, the $z$-coordination of the metal atoms can be determined, providing valuable information for understanding the catalytic properties of supported SACs. Adapted from Ref. [61]. 
hours. With high levels of Ir loading onto the flat and smooth \{10-10\} nanoscale facets of the $\mathrm{ZnO}$ nanowires the exact 3D position of the Ir atoms can be directly visualized by obtaining images of the different orientations of the $\mathrm{ZnO}$ nanowires [61]. The ac-STEM-HAADF image of Fig. 8 unambiguously shows that the $\mathrm{Ir}_{1}$ atoms were embedded onto the $\mathrm{ZnO}\{10-10\}$ surfaces as if they replaced the $\mathrm{Zn}$ cation atoms. There are no obvious association of the Ir atoms with the $\mathrm{ZnO}\{10-10\}$ surface steps. The Ir atoms did not diffuse into the subsurface regions of the $\mathrm{ZnO}$ even after prolonged calcination at temperatures $>500{ }^{\circ} \mathrm{C}$. Analyses of such ac-STEM-HAADF images obtained with different orientations of well-defined support nanostructures can provide valuable information on the surface structure of the support as well as the exact position of the surface adsorbed metal atoms. It is expected that ac-STEM techniques will become even more powerful for characterizing single metal atoms supported on nanostructured supports such as 2D or shape controlled metal oxides.

\section{Summary and perspective}

In this perspective, we summarized the capability of ac-STEM-HAADF imaging and the application of this powerful technique to characterizing supported single metal atoms. The sub-angstrom resolution and the atomic number sensitivity of the STEM-HAADF imaging technique make it possible not only to examine the dispersion of supported single metal atoms but also to obtain their lateral coordinate information with respect to the surface structure of the crystalline support. Such atomic scale data on the coordinates of the supported single metal atoms can be directly used to establish models for DFT calculations of the catalytic processes of SACs. The STEM-HAADF imaging technique has proved to be indispensable for optimizing synthesis protocols, studying the stability of SACs, and probing the nature of catalytically active centers. The major challenge to the broad applications of the STEM associated techniques is that of the electron-beam induced effects. For TEM imaging, low dose method, coupled with the recently developed highly efficient ultrafast cameras and image processing, has been effectively utilized to image electron-beam sensitive materials such as zeolites and metal organic frameworks [62]. Further technical development is needed to alleviate the electron-beam induced effects in STEM instrument to the degree that localized EDS and EELS spectroscopy techniques can be applied to reliably analyze the composition and oxidation states of supported single metal atoms. Information on the isolated metal atoms from such localized spectroscopy techniques, especially with monochromated high energy-resolution EELS, should be extremely valuable for understanding the fundamental catalytic processes of supported SACs.

The rapid development of gas/liquid sample holders or environmental EM chambers, the use of ultra-stable sample heating holders, the use of pulsed electron sources, the development of new electron detectors, and the availability of atomic resolution cryo-EMs can significantly enhance our capability of characterizing heterogeneous catalysts, especially supported single-atom catalysts. It is expected that the combination of
STEM techniques with other broad beam spectroscopy techniques such as X-ray absorption spectroscopy and IR spectroscopy will help us understand the catalytic nature of supported single metal atoms, develop robust SACs for practical applications, and connect heterogeneous catalysis to homogeneous catalysis.

\section{Acknowledgments}

This work was funded by the National Science Foundation under CHE-1465057 and the National Key Basic Research Program of China (973) (2013CB933104). The author acknowledges the use of the facilities in the John M. Cowley Center for High Resolution Electron Microscopy at Arizona State University. The author acknowledges his collaborators Dr. Lawrence F. Allard of Oak Ridge National Laboratory, Professors Tao Zhang, Aiqing Wang and Weixue Li of the Dalian Institute of Chemical Physics of the Chinese Academy of Sciences, Professor Jun Li of Tsinghua University, and Drs. Botao Qiao, Yang Lou, Jia $\mathrm{Xu}$, and Sibin Duan as well as Mr. Yafeng Cai.

Jingyue (Jimmy) Liu

a Department of Physics, Arizona State University, Tempe, Arizona 85287, United States

b State Key Laboratory of Catalysis, Dalian Institute of Chemical Physics, Chinese Academy of Sciences, Dalian 116023, Liaoning, China

E-mail: Jingyue.Liu@asu.edu

Received 23 July 2017

Published 5 September 2017

DOI: 10.1016/S1872-2067(17)62900-0

\section{References}

[1] A. V. Crewe, J. Wall, J. Langmore, Science, 1970, 168, 1338-1340.

[2] J. Wall, J. Langmore, M. Isaacson, A. V. Crewe, Proc. Natl. Acad. Sci. USA, 1974, 71, 1-5.

[3] M. Isaacson, D. Kopf, M. Utlaut, N. W. Parker, A. V. Crewe, Proc. Natl. Acad. Sci. USA, 1977, 74, 1802-1806.

[4] M. M. J. Treacy, A. Howie, C. J. Wilson, Philos. Mag. A, 1978, 38, 569-585.

[5] A. Howie, J. Microsc., 1979, 117, 11-23.

[6] M. M. J. Treacy, S. B. Rice, J. Microsc., 1989, 156, 211-234.

[7] S. B. Rice, J. Y. Koo, M. M. Disko, M. M. J. Treacy, Ultramicroscopy, 1990, 34, 108-118.

[8] J. Liu, J. M. Cowley, Ultramicroscopy, 1990, 34, 119-128.

[9] J. Liu, Microsc. Microanal., 2004, 10, 55-76.

[10] J. Liu, J. Electron Microsc., 2005, 54, 251-278.

[11] J. Liu, ChemCatChem, 2011, 3, 934-948.

[12] P. D. Nellist, S. J. Pennycook, Science, 1996, 274, 413-415.

[13] B. T. Qiao, A. Q. Wang, X. F. Yang, L. F. Allard, Z. Jiang, Y. T. Cui, J. Y. Liu, J. Li, T. Zhang, Nat. Chem., 2011, 3, 634-641.

[14] J. Lu, C. Aydin, N. D. Browning, B. C. Gates, Angew. Chem. Int. Ed., 2012, 51, 5842-5846.

[15] J. Lin, A. Q. Wang, B. T. Qiao, X. Y. Liu, X. F. Yang, X. D. Wang, J. X. Liang, J. Li, J. Y. Liu, T. Zhang, J. Am. Chem. Soc., 2013, 135, 15314-15317.

[16] M. Yang, L. F. Allard, M. Flytzani-Stephanopoulos, J. Am. Chem. Soc., 2013, 135, 3768-3771. 


\section{Graphical Abstract}

Chin. J. Catal., 2017, 38: 1460-1472 doi: 10.1016/S1872-2067(17)62900-0

\section{Aberration-corrected scanning transmission electron microscopy in single-atom catalysis: Probing the catalytically active centers}

Jingyue (Jimmy) Liu *

Arizona State University, United States;

Dalian Institute of Chemical Physics, Chinese Academy of Sciences, China
Aberration-corrected scanning transmission electron microscopy techniques are indispensable for understanding catalysis by supported single metal atoms and for developing practical single-atom catalysts for energy and chemical transformations.

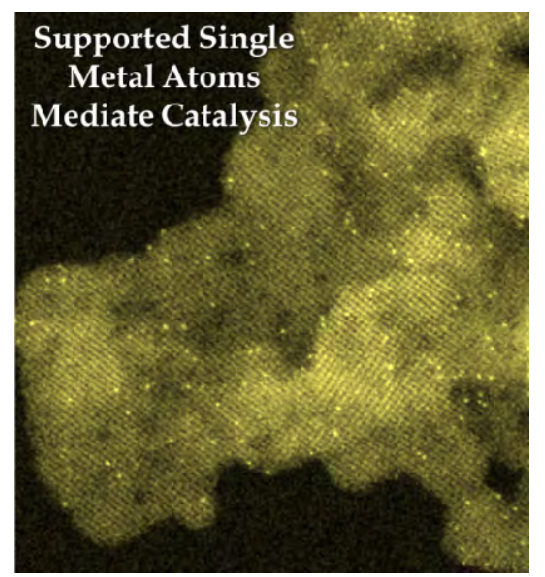

[17] M. Moses-DeBusk, M. Yoon, L. F. Allard, D. R. Mullins, Z. L. Wu, X. F. Yang, G. Veith, G. M. Stocks, C. K. Narula, J. Am. Chem. Soc., 2013, 135, 12634-12645.

[18] H. S. Wei, X. Y. Liu, A. Q. Wang, L. L. Zhang, B. T. Qiao, Y. F. Yang, Y. Q. Huang, S. Miao, J. Y. Liu, T. Zhang, Nat. Commun., 2014, 5, 5634.

[19] B. T. Qiao, J. X. Liang, A. Q. Wang, C. Q. Xu, J. Li, T. Zhang, J. Y. Liu, Nano Res., 2015, 8, 2913-2924.

[20] M. Yang, J. L. Liu, S. Lee, B. Zugic, J. Huang, L. F. Allard, M. Flytzani-Stephanopoulos, J. Am. Chem. Soc., 2015, 137, 3470-3473.

[21] J. Y. Liu, ACS Catal., 2017, 7, 34-59.

[22] J. D. Kistler, N. Chotigkrai, P. Xu, B. Enderle, P. Praserthdam, C. Y. Chen, N. D. Browning, B. C. Gates, Angew. Chem. Int. Ed., 2014, 53, 8904-8907.

[23] J. C. Matsubu, V. N. Yang, P. Christopher, J. Am. Chem. Soc., 2015, 137, 3076-3084.

[24] M. Haider, H. Rose, S. Uhlemann, E. Schwan, B. Kabius, K. Urban, Ultramicroscopy, 1998, 75, 53-60.

[25] M. Haider, S. Uhlemann, E. Schwan, H. Rose, B. Kabius, K. Urban, Nature, 1998, 392, 768-769.

[26] O. L. Krivanek, N. Dellby, A. J. Spence, R. A. Camps, L. M. Brown, Electron Microsc. Anal., 1997, 153, 35-40.

[27] O. L. Krivanek, N. Dellby, A. R. Lupini, Ultramicroscopy, 1999, 78, 1-11.

[28] O. L. Krivanek, M. F. Chisholm, V. Nicolosi, T. J. Pennycook, G. J. Corbin, N. Dellby, M. F. Murfitt, C. S. Own, Z. S. Szilagyi, M. P. Oxley, S. T. Pantelides, S. J. Pennycook, Nature, 2010, 464, 571-574.

[29] (a) J. Liu, Microsc. Microanal., 2007, 13 (Suppl 2), 544-545; (b) J. Liu, J. Wang, L. F. Allard, Microsc. Microanal., 2008, 14 (Suppl 2), 262-263; (c) J. Liu, L. F. Allard, Proc 21st NAM Meeting, 2008; (d) J. Liu, L. F. Allard, Microsc. Microanal., 2009, 15 (Suppl 2), 1486-1487; (e) J. Liu, J. Braddock-Wilking, L. F. Allard, Microsc. Microanal., 2009, 15 (Suppl 2), 688-689; (f) J. Liu, L. F. Allard, Microsc. Microanal., 2011, 17 (Suppl 2), 1282-1283.

[30] L. F. Allard, A. Borisovich, W. Deng, R. Si, M. Flytzani-Stephanopoulos, S. H. Overbury, J. Electron Microsc., 2009, 58, 199-212.

[31] M. Flytzani-Stephanopoulos, Acc. Chem. Res., 2014, 47, 783-792.

[32] B. T. Qiao, J. X. Liu, Y. G. Wang, Q. Lin, X. Liu, A. Wang, J. Li, T. Zhang, J. Liu, ACS Catal., 2015, 5, 6249-6254.
[33] Y. Pan, N. Nilius, H. J. Freund, J. Paier, C. Penschke, C. J. Sauer, Phys. Rev. Lett., 2013, 111, 206101/1-206101/5.

[34] G. Pacchioni, H. Freund, Chem. Rev., 2012, 113, 4035-4072.

[35] G. Kyriakou, M. B. Boucher, A. D. Jewell, E. A. Lewis, T. J. Lawton, A. E. Baber, H. L. Tierney, M. Flytzani-Stephanopoulos, E. C. H. Sykes, Science, 2012, 335, 1209-1212.

[36] Y. Zhai, D. Pierre, R. Si, W. Deng, P. Ferrin, A. U. Nilekar, G. Peng, J. A. Herron, D. C. Bell, H. Saltsburg, M. Mavrikakis, M. Flytzani-Stephanopoulos, Science, 2010, 329, 1633-1636.

[37] Y. Lou, J. Liu, Ind. Eng. Chem. Res., 2017, 56, 6916-6925.

[38] J. M. Thomas, R. Raja, D. W. Lewis, Angew. Chem. Int. Ed., 2005, 44, 6456-6482.

[39] J. D. Kistler, N. Chotigkrai, P. Xu, B. Enderle, P. Praserthdam, C. Y. Chen, N. D. Browning, B. C. Gates, Angew. Chem. Int. Ed., 2014, 53, 8904-8907.

[40] P. Xu, J. Lu, C. Aydin, L. M. Debefve, N. D. Browning, C. Y. Chen, B. C. Gates, Microporous Mesoporous Mater., 2015, 213, 95-99.

[41] D. H. Deng, X. Q. Chen, L. Yu, X. Wu, Q. F. Liu, Y. Liu, H. X. Yang, H. F. Tian, Y. F. Hu, P. P. Du, R. Si, J. H. Wang, X. J. Cui, H. B. Li, J. P. Xiao, T. Xu, J. Deng, F. Yang, P. N. Duchesne, P. Zhang, J. G. Zhou, L. T. Sun, J. Li, X. L. Pan, X. H. Bao, Sci. Adv., 2015, 1, e1500462/1-e1500462/9.

[42] X. G. Li, W. T. Bi, L. Zhang, S. Tao, W. S. Chu, Q. Zhang, Y. Luo, C. Z. Wu, Y. Xie, Adv. Mater., 2016, 28, 2427-2431.

[43] J. Deng, H. B. Li, J. P. Xiao, Y. C. Tu, D. H. Deng, H. X. Yang, H. F. Tian, J. Q. Li, P. J. Ren, X. H. Bao, Energy Environ. Sci., 2015, 8, 1594-1601.

[44] Y. J. Gong, Z. Liu, A. R. Lupini, G. Shi, J. H. Lin, S. Najmaei, Z. Lin, A. L. Elías, A. Berkdemir, G. You, H. Terrones, M. Terrones, R. Vajtai, S. T. Pantelides, S. J. Pennycook, J. Lou, W. Zhou, P. M. Ajayan, Nano Lett., 2014, 14, 442-449.

[45] J. Perez-Ramirez, G. Vile, D. Albani, M. Nachtegaal, Z. Chen, D. Dontsova, M. Antonietti, N. Lopez, Angew. Chem. Int. Ed., 2015, 54, 11265-11269.

[46] L. L. Zhang, A. Q. Wang, W. T. Wang, Y. Q. Huang, X. Y. Liu, S. Miao, J. Y. Liu, T. Zhang, ACS Catal, 2015, 5, 6563-6572.

[47] R. F. Egerton, Microsc. Microanal., 2013, 19, 479-486.

[48] R. F. Egerton, Ultramicroscopy, 2014, 145, 85-93.

[49] L. Zhao, R. He, K. T. Rim, T. Schiros, K. S. Kim, H. Zhou, C. Gutiérrez, 
S. P. Chockalingam, C. J. Arguello, L. Pálová, D. Nordlund, M. S. Hybersen, D. R. Reichman, T. F. Heinz, P. Kim, A. Pinczuk, G. W. Flynm, A. N. Pasupathy, Science, 2011, 333, 999-1003.

[50] W. Zhou, M. D. Kapetanakis, M. P. Prange, S. T. Pantelides, S. J. Pennycook, J. C. Idrobo, Phys. Rev. Lett., 2012, 109, 206803/1-206803/5.

[51] J. H. Warner, Y. C. Lin, K. He, M. Koshino, K. Suenaga, ACS Nano, 2014, 8, 11806-11815.

[52] O. L. Krivanek, T. C. Lovejoy, N. Dellby, T. Aoki, R. W. Carpenter, P. Rez, E. Soignard, J. Zhu, P. E. Batson, M. J. Lagos, R. F. Egerton, P. A., Crozier. Nature, 2014, 514, 209-212.

[53] M. J. Lagos, A. Trügler, U. Hohenester, P. E. Batson. Nature, 2017, 543, 529-532.

[54] P. A. Midgley, E. P. W. Ward, A. B. Hungria, J. M. Thomas, Chem. Soc. Rev., 2007, 36, 1477-1494.
[55] H. Friedrich, P. E. de Jongh, A. J. Verkleij, K. P. de Jong, Chem. Rev., 2009, 109, 1613-1629.

[56] P. A. Midgley, R. E. Dunin-Borkowski, Nat. Mater., 2009, 8, 271-280.

[57] L. F. Allard, S. H. Overburg, W. C. Bigelow, M. B. Katz, D. P. Nackashi, J. Damiano, Microsc. Microanal., 2012, 18, 656-666.

[58] L. F. Allard, S. Duan, J. Liu, Microsc. Microanal., 2016, 22(S2), 876-877.

[59] E. D. Boyes, P. L. Gai, Comp. Rend. Phys., 2014, 15, 200-213.

[60] P. L. Gai, L. Lari, M. R. Ward, E. D. Boyes, Chem. Phys. Lett., 2014, 592, 355-359.

[61] J. Xu, Y. Song, H. Wu, J. Liu, Microsc. Microanal., 2017, 23(S1), 484-485.

[62] Y. H. Zhu, Y. Pan, Z. P. Lai, C. E. Hsiung, K. Yao, I. Pinnau, Y. Han, J. Ciston, B. Zheng, X. Miao, R. Sougrat, C. Czarnik, M. Pan, Nat. Mater., 2017, 16, 532-536.

\title{
球差校正扫描透射电子显微镜(ac-STEM)探测单原子催化中的活性中心
}

\author{
刘景月 $\mathrm{a,b}, *$ \\ a亚利桑那州立大学物理系, 亚利桑那州坦佩 85287, 美国 \\ b 中国科学院大连化学物理研究所催化重点国家实验室, 辽宁大连 116023, 中国
}

\begin{abstract}
摘要: 近年来, “单原子催化” 逐渐得到人们的认可与关注, 成为催化领域新的前沿与热点之一. 高分辨扫描透射电子显微 镜(STEM), 特别是带有球差校正(ac)的高角环形暗场扫描透射电子显微镜(ac-STEM-HAADF)是唯一能够直接 “看到” 催化 剂中单个原子的工具. 在单原子催化的发现、单原子催化剂的开发、催化剂制备的优化、以及单原子催化机理的理解方 面具有举足轻重的、甚至是不可替代的作用. 本文首先简述了早期的高分辨透射电子显微镜(HRTEM)逐步发展成目前的 ac-STEM-HAADF的过程以及过程中各种电镜在单原子观察中的功能. 然后综述了ac-STEM在单原子成像、检测单原子分 散度及改进催化剂制备参数、检测单原子催化剂中单原子的配位及催化活性中心中的应用及其机遇与挑战.

1990年代后期在透镜相差校正领域技术的突破使STEM的分辨率显著提高, 因此STEM的单原子成像灵敏度得到明显 改善, 因而能够对实用催化剂中的单个重原子进行具有很好相祄度的常规检测. 2000年代末期刘景月课题组开始进行系 统的单原子催化剂合成与表征, 表明ac-STEM-HAADF毫无疑问地能够进行实用催化剂中的单个金属原子的常规检测. 随 后与张涛课题组合作启动了一项采用简便可放大的共沉淀法制备单原子催化剂的项目并取得成功. 相比于光谱表征提供 样品平均与整体信息, 电子显微镜能够提供具有空间分辨的局部信息. 对于含有从微米到纳米不同级别不均一性的多相 催化剂而言, 该技术尤其具有价值.

除了能够提供负载金属单原子的空间分布与分散度信息外, 如果载体是晶体, ac-STEM-HAADF还能够提供每个金属 原子相对于载体表面的空间配位信息. 而对于非晶载体例如活性炭、无定型氧化硅等, 则以上信息全部丢失, 只能提供负 载金属原子的空间分布与分散度信息.

电子显微镜面临的最主要挑战在于电子束辐射效应: 高能电子与样品作用会导致表面金属原子的迁移、表面官能团 的消失或改变以及损坏样品等; 还会引起环境电镜中气体的离子化. 对于大多数催化剂而言, 采用低能量电子束能够有效 减少电子束辐射影响. 由于电子束影响, 目前还难以对单原子催化剂进行X射线能量色散谱和电子能量损失谱的常规检测, 需要开发更加高效的检测器. 此外, 电子显微镜只提供三维材料的二维投影, 因此在成像过程中不但三维信息丢失, 而且 对二维投影的解释也具有挑战, 因此需要开发具有原子分辨率的三维成像技术. 为了更好理解催化剂的制备和催化反应 过程, 也亟需发展具有单原子分辨率的环境电镜.
\end{abstract}

关键词：单原子催化; 球差校正扫描透射电子显微镜; 探测; 活性中心

收稿日期: 2017-07-23. 接受日期: 2017-08-21. 出版日期: 2017-09-05.

*通讯联系人. 电子信箱: Jingyue.Liu@asu.edu

本文的英文电子版由Elsevier出版社在ScienceDirect上出版(http://www.sciencedirect.com/science/journal/18722067). 